\title{
COVID-19 in patients undergoing chronic kidney replacement therapy and kidney transplant recipients in Scotland: findings and experience from the Scottish renal registry
}

Samira Bell ${ }^{1,2^{*}}$ (D) Jacqueline Campbell ${ }^{2}$, Jackie McDonald ${ }^{2}$, Martin $\mathrm{O}^{\prime}$ Neill $^{2}$, Chrissie Watters ${ }^{2}$, Katharine Buck ${ }^{3}$, Zoe Cousland ${ }^{4}$, Mark Findlay ${ }^{5}$, Nazir I. Lone ${ }^{6}$, Wendy Metcalfe ${ }^{7}$, Shona Methven ${ }^{8}$, Robert Peel ${ }^{9}$, Alison Almond ${ }^{10}$, Vinod Sanu ${ }^{11}$, Elaine Spalding ${ }^{12}$, Peter C. Thomson ${ }^{5}$, Patrick B. Mark ${ }^{5}$, Jamie P. Traynor ${ }^{2,5}$ and on behalf of the Scottish Renal Registry

\begin{abstract}
Background: Infection with the severe acute respiratory coronavirus 2 (SARS-CoV-2) has led to a worldwide pandemic with coronavirus disease 2019 (COVID-19), the disease caused by SARS-CoV-2, overwhelming healthcare systems globally. Preliminary reports suggest a high incidence of infection and mortality with SARS-CoV-2 in patients receiving kidney replacement therapy (KRT). The aims of this study are to report characteristics, rates and outcomes of all patients affected by infection with SARS-CoV-2 undergoing KRT in Scotland.
\end{abstract}

Methods: Study design was an observational cohort study. Data were linked between the Scottish Renal Registry, Health Protection Scotland and the Scottish Intensive Care Society Audit Group national data sets using a unique patient identifier (Community Health Index (CHI)) for each individual by the Public Health and Intelligence unit of Public Health, Scotland. Descriptive statistics and survival analyses were performed.

Results: During the period 1st March 2020 to 31st May 2020, 110 patients receiving KRT tested positive for SARS-CoV-2 amounting to $2 \%$ of the prevalent KRT population. Of those affected, 86 were receiving haemodialysis or peritoneal dialysis and 24 had a renal transplant. Patients who tested positive were older and more likely to reside in more deprived postcodes. Mortality was high at $26.7 \%$ in the dialysis patients and $29.2 \%$ in the transplant patients.

\footnotetext{
* Correspondence: s.t.bell@dundee.ac.uk

'Division of Population Health and Genomics, School of Medicine, University of Dundee, Dundee DD1 9SY, UK

${ }^{2}$ The Scottish Renal Registry, Scottish Health Audits, Public Health \&

Intelligence, Information Services, Meridian Court, 5 Cadogan Street, Glasgow G2 6QE, UK

Full list of author information is available at the end of the article
}

(c) The Author(s). 2020 Open Access This article is licensed under a Creative Commons Attribution 4.0 International License, which permits use, sharing, adaptation, distribution and reproduction in any medium or format, as long as you give appropriate credit to the original author(s) and the source, provide a link to the Creative Commons licence, and indicate if changes were made. The images or other third party material in this article are included in the article's Creative Commons licence, unless indicated otherwise in a credit line to the material. If material is not included in the article's Creative Commons licence and your intended use is not permitted by statutory regulation or exceeds the permitted use, you will need to obtain permission directly from the copyright holder. To view a copy of this licence, visit http://creativecommons.org/licenses/by/4.0/. The Creative Commons Public Domain Dedication waiver (http://creativecommons.org/publicdomain/zero/1.0/) applies to the data made available in this article, unless otherwise stated in a credit line to the data. 
(Continued from previous page)

Conclusion: The rate of detected SARS-CoV-2 in people receiving KRT in Scotland was relatively low but with a high mortality for those demonstrating infection. Although impossible to confirm, it appears that the measures taken within dialysis units coupled with the national shielding policy, have been effective in protecting this population from infection.

Keywords: COVID-19, Kidney replacement therapy, Epidemiology, Registry

\section{Background}

Infection with the severe acute respiratory coronavirus 2 (SARS-CoV-2) has led to a worldwide pandemic with COVID-19, the disease caused by SARS-CoV-2, overwhelming healthcare systems globally [1]. The clinical course of the disease varies significantly from asymptomatic disease to multi-organ failure and death [2]. Patients receiving kidney replacement therapy (KRT) are a vulnerable population as those receiving dialysis are usually older with significant co-morbidity, have impaired immune responses [3] and require regular attendance at a healthcare facility which further increases their risk of exposure. Patients who have a kidney transplant are presumed to be at high risk due to their obligate immunosuppressive medications. There have been a number of published recommendations on how to mitigate spread of SARS-CoV-2 in dialysis units [4-8]. There is, however, a lack of data regarding clinical course and outcome in patients receiving KRT following infection with SARS-CoV-2. Furthermore, early reports suggest that case fatality in dialysis patients if infected is high with reports suggesting fatality in the order of $5-20 \%$ compared to $1-2 \%$ in patients not requiring KRT [9-11]. Patients requiring KRT often have multiple comorbid conditions which are widely documented as increasing the risk of death from COVID-19 such as older age, chronic conditions such as diabetes mellitus and hypertension [12, 13] but may be at additional risk due to the relatively impaired immune response associated with kidney failure.

The first case of SARS-CoV-2 infection in Scotland was confirmed on 1st March 2020, although subsequent data suggest that the virus had been in circulation for weeks prior to this [14]. On 24th March, as part of the Scottish Government response to the pandemic, certain groups of patients were identified as the most vulnerable to developing severe illness and advised to "shield" meaning they were advised to remain at home and minimise all non-essential contact with other members of their household. This "shielded" category included kidney transplant recipients (KTR) from the outset but Scottish (and United Kingdom) government advice was not applied to dialysis patients until 28th April 2020. However, most renal units commenced implementation of PPE protocols prior to government advice. Delivering care to these patients whilst minimising risk of exposure and preventing transmission of infection to these vulnerable patient groups posed significant challenges to renal units across the country.

The aims of this study are to report characteristics, rates and outcomes of all patients affected by infection with SARS-CoV-2 undergoing chronic KRT including KTRs in Scotland.

\section{Methods}

Data sources

Study design was an observational cohort study. The following national data sets were linked using a unique patient identifier (Community Health Index (CHI)) for each individual by Public Health and Intelligence unit of Public Health, Scotland. The Scottish Renal Registry is a national registry of all patients receiving KRT for end stage renal disease (ESRD) in Scotland (haemodialysis, peritoneal dialysis and transplant). It was established in 1991 with data backfilled to 1960 from European Renal Association-European Dialysis and Transplant Association (ERA-EDTA) with the first patient dialysed for ESRD in Scotland in 1960. The Scottish Renal Registry has $100 \%$ unit and patient coverage. Data held by the registry include patient demographics including historical postcodes (for calculating Scottish Index of Multiple Deprivation), full KRT history (for ESRD), date and cause of death (using ERA-EDTA codes), primary renal diagnosis (using ERA-EDTA codes) and monthly linkage with National Health Service Blood and Transplant (NHS BT) for transplant status. The primary renal diagnosis groupings used in the analyses is described in the Scottish Renal Registry website [15]. The Scottish Government provide online calculators allowing use of patient postcode to generate divisions of socioeconomic deprivation, the Scottish Index of Multiple Deprivation (SIMD) [16]. Using patient postcode, deprivation quintiles were calculated and categorized into most deprived quintiles with 1 corresponding to most deprived and 5 as least deprived. Data on testing for COVID-19 were obtained from Health Protection Scotland with date of test and result reported. Data on admission to intensive care units were obtained from the Scottish Intensive Care Society Audit Group (SICSAG). The Scottish Intensive Care Society Audit Group (SICSAG) has maintained a national database of patients admitted to adult general Intensive Care Units (ICU) in Scotland since 
1995 [17]. Detailed information is produced on the management of critically ill or injured patients. All general intensive care units (ICU) and combined ICU/high dependency units (HDU) collect data. During this period, there was no limitation of access to ICU admission for patients with comorbid conditions, with patients admitted to ICU if clinicians felt it was clinically appropriate.

Testing for SARS-CoV-2 was carried out by real-time polymerase chain reaction (RT-PCR) on a combined nasal and pharyngeal throat swab only in symptomatic patients or endotracheal aspirate for those ventilated in ICU. If a patient has been tested multiple times, the date of the first positive or first negative test was used. If a patient has tested positive, then subsequently tested negative they would only appear in the positive cohort.

\section{Statistical analysis}

Data were linked and analysed by a Public Health Intelligence analyst at Public Health Scotland. Baseline characteristics were displayed as mean and standard deviation (SD) or median and interquartile range (IQR) for continuous variables depending on normality. T-tests and chi-squared tests were utilised to examine differences in parameters between those who died and those were still alive as of 31st May 2020. Categorical variables were displayed as number and percentage. Logistic regression was performed with SARS-Cov- 2 positivity as a binary outcome. Crude survival was measured at 30 days since first SARS-Cov-2 test using Kaplan Meier survival analyses. Cox proportional hazards models were performed to examine predictors of death. All analyses were performed using R Studio 3.5.1 [18].

\section{Results}

\section{Scottish Renal Registry}

The Scottish renal registry collates data from all 9 adult renal units in Scotland and 28 satellite haemodialysis units (Fig. 1) serving a population of 5.4 million. On 31st May 2020, there were 5475 prevalent patients in Scotland receiving KRT. Of these, 1983 were on haemodialysis with 206 peritoneal dialysis and 3286 with functioning kidney transplants.

\section{Characteristics of SARS-CoV-2 positive patients}

As of 31st May 2020, 876 patients receiving KRT had been tested for SARS-CoV-2 infection. This represents $16 \%$ of the total number of 5475 receiving KRT on 31st May 2020. One hundred and ten were positive which amounts to $2 \%$ of the prevalent KRT population. Of those affected, 86 were receiving haemodialysis or peritoneal dialysis and 24 had a renal transplant. Figure 2 shows the distribution of those individuals who tested positive for SARS-CoV-2 by geographical location relative to the whole KRT population. In keeping with the national figures related to the general population, the majority of positive tests were within the central belt of Scotland. Figure 3a shows the number of positive cases by week with a peak in positive cases between the 30th of March and 13th of April. This contrasts with data for general population across Scotland where the number of new cases only started to fall during week beginning 27th April (Fig. 3b). There were 986 tests performed in 876 patients until the 31st May with 110 (11.2\%) testing positive. The cumulative number tested and the proportion of positive cases over time is shown in Figs. 4a and b with a sharp increase in testing over the time period examined. Characteristics of the kidney replacement population and those who tested positive for SARSCoV-2 are shown in Table 1. Mean age of those tested positive was 64.3 years old (SD 14.4) with a primary renal diagnosis of diabetes in $33.7 \%$ and median dialysis vintage of 4 years (IQR $1-8$ years). Of the 24 patients who had a functioning kidney transplant when tested positive for SARS-CoV-2, the majority of the transplants $(n=14)$ were performed over 10 years ago with $8 \%(n=$ 2) cases recorded had a transplant less than 2 years ago. $65 \%$ of those who tested positive lived in the most deprived areas (SIMD 1 or 2). 98 of 110 who tested positive were of white ethnicity. We do not have data on the ethnicity of the entire KRT population. Using logistic regression, increasing age at time of infection OR 1.038 (95\%CI 1.023-1.053), $p<0.001$ and social deprivation OR 0.778 (95\% CI $0.673-0.899$ ), $p=0.001$ (with every quintile increase in in SIMD) were predictors of testing positive for all those on KRT on 31 May 2020 registered on the SRR.

\section{Patient outcomes}

As of the 31st May 2020, 30 (28.2\%) of the patients who tested positive for SARS-CoV-2 infection died. Twenty three $(26.7 \%)$ of the 86 patients treated with haemodialysis or peritoneal dialysis who tested positive died. Seven (29.2\%) of the 24 transplant patients who tested positive died. Mean age of death was higher in those who died compared with those who were still alive as of 31st May 2020 (69 years (SD 11.6) vs. 62 years (SD 8.5), $p=$ 0.0182 ). There was no difference in median dialysis vintage of those who died compared with those who were still alive as of 31st May 2020: 4 years (IQR 1-12 years) vs 4 years (IQR $1-7$ years, $p=0.34$ ). One third of patients who died had a primary renal diagnosis of diabetic nephropathy. Figure 5 shows the total number of deaths in the KRT therapy population in Scotland from 1st January 2020 to 31st May 2020 with those who had COVID-19 identified as a primary cause of death on the death certificate. Figure 6 shows the number of deaths from 1st January to 31st May each year from 2017 to 2020. Deaths in 2020 not related to COVID-19 are also 


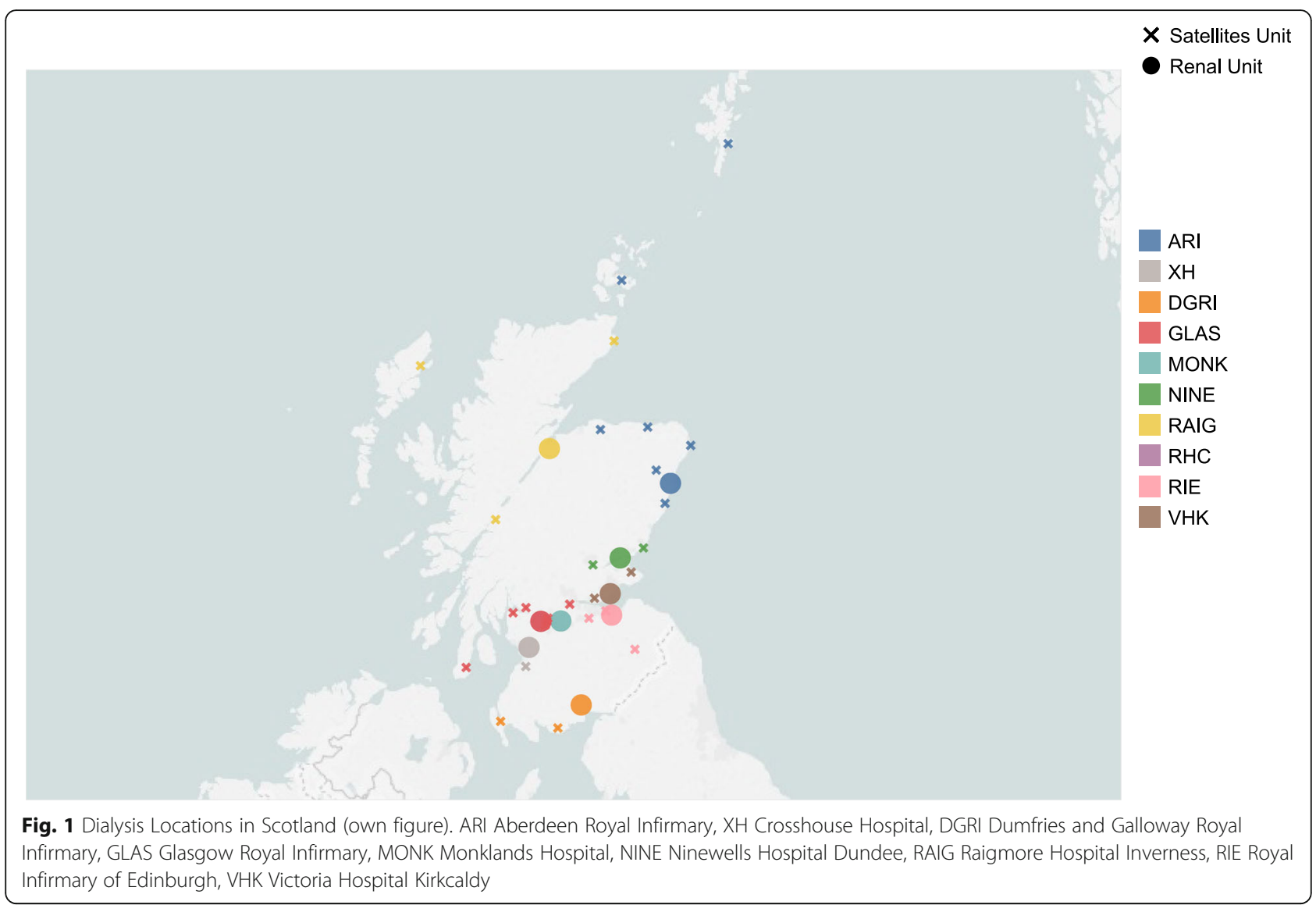

shown. Both Figs. 5 and 6 demonstrate an increase in deaths occurring in April compared with other months. Figure 5 in particular highlights that a large proportion (35\%) of the deaths occurring in April were related to COVID-19. Crude thirty day survival is shown in Fig. 7a and b. There was 91\% (95\% CI 89-94) 30 day survival in those to tested negative compared with $71 \%$ (95\% CI 63-80) 30 day survival in those who tested positive. Increasing age was associated with mortality in cox proportional hazards model with an increase of hazard ratios for death of 1.028 (95\% CI 1.001 to $1.055, p=$ 0.038 ) for every year of age.

\section{Intensive care admission}

During the period from 1st March 2020 to 31st May 2020 there were a total of 118 patients registered on the SRR who were admitted to an ICU/HDU in Scotland. Of these $13(13 \%)$ patients had tested positive for SARSCoV-2 infection. $92.3 \%$ of those with SARS-CoV-2 were discharged alive from ICU compared with $94.1 \%$ who were not tested/tested negative. However, of those who were discharged alive from ICU/HDU $41.6 \%$ of those with a positive SARS-CoV-2 test died within 7 days of being discharged compared to $6.94 \%$ of those who did not have a positive test result. Details of ICU/ HDU admission are shown in Table 2.

\section{Discussion}

As of May 31st 2020, SARS-CoV-2 was detected in 2\% of the prevalent Scottish KRT population (3.9\% of the prevalent dialysis population and $0.7 \%$ of the prevalent transplant population). Mortality was high at $26.7 \%$ in the dialysis patients and $29.2 \%$ in the transplant patients. Patients who tested positive were more likely to be older and from more deprived postcodes. Increasing age was associated with mortality.

During the early stages of the pandemic, advice about personal protective equipment (PPE) was subject to a number of changes mainly in response to guidance issued by UK government for use of PPE in the healthcare environment. On 2nd April 2020, the UK and Scottish governments issued new PPE guidelines for patients and staff in secondary care and these in turn informed the UK Renal Association recommendations for PPE in dialysis units $[8,19]$. The guidance has largely remained unchanged since and essentially advocates that staff use face masks (one per session) and single use aprons and gloves with all patients, including those where COVID was not suspected. After discussion with local 


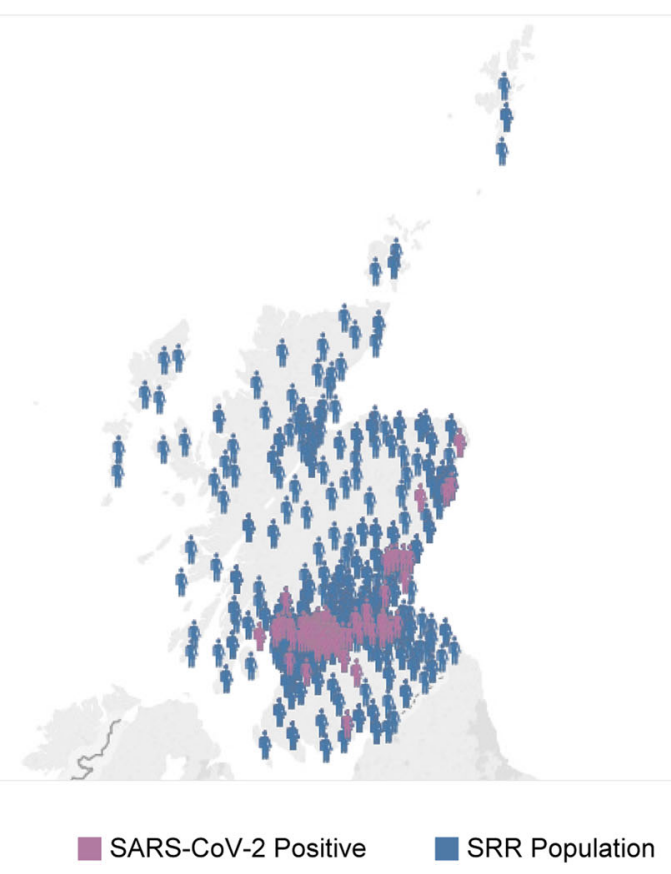

Fig. 2 Geographical output of SARS-CoV-2 in KRT patients up to 31 May 2020 (own figure)

microbiology/infectious disease/infection control teams, most units implemented these changes in renal dialysis units very quickly with all units following this guidance by 6th April 2020. Two units adopted this approach before the government advice with the earliest adoption of PPE on 13th March 2020.

All dialysis patients now wear a mask for travelling into dialysis and during the session. Patients are given a mask to use for next session. Patients are advised to minimise time spent eating or drinking i.e. time with mask off. Waiting areas are a potential challenge as in some units these are very small and cannot facilitate adequate social distancing. Approaches to address this includes staggering start times for dialysis and asking patients to wait in their transport vehicle until the staff are ready to commence their dialysis session. All patients have individual transport except a few cases where following an adequate risk assessment, two patients share an ambulance but wear masks. Individualised transport has only been possible whilst there is reduced demand from other specialties which have put non-essential services on hold or at significant cost to the units by providing single occupancy taxi for transport. Most units have, or are actively making plans for, the time when individual transport is no longer available or manageable. All units measure patient temperature on arrival. The threshold for action varies between temperature of $37.8^{\circ}$ and $38.0^{\circ}$ degrees with the exception of one satellite unit which has a threshold of $37.0^{\circ}$. Once reached, this threshold triggers testing for SARS-COV2 and dialysis in isolation. All units have taken measures to minimise visits to hospital for people with a functioning transplant. Clinical records are reviewed in advance of clinics. Unless a face-to-face consultation is considered necessary, alternative options have included telephone consultations, blood tests only and increased time between clinics. Phone consultations are provided mostly by medical staff but some units include transplant nurse specialists. Bloods have been taken in a variety of settings including newly set up 'bloods only' clinics, bespoke mobile phlebotomy service, existing community hubs or in coordination with primary care.

There is currently a lack of data on how SARS-CoV-2 affects patients receiving haemodialysis. Early reports from Wuhan suggested a milder disease in these patients [20]. However, more recent reports are suggesting worse outcomes. The Lombardy and Brescia regions of Italy were severely affected by COVID-19. An observational study from 4 dialysis centres in the Brescia region of Italy showed that $95(15 \%)$ of 643 haemodialysis patients tested positive for COVID-19 with 27 (29\%) of these dying [21]. A further observational study from a haemodialysis centre in Spain found that 36 patients out of 282 patients tested positive for COVID-19 and 11 (30.5\%) of these patients subsequently died. Dialysis vintage was associated with mortality in this cohort of patients [22]. Similarly, a study of 59 consecutive patients testing positive from Columbia University in the United States found a mortality rate of 31\% (18 patients) [11]. A report from the COVID19 registry of the Spanish Society of Nephrology reported a mortality of $23 \%$ in 868 patients with SARS-CoV-2 


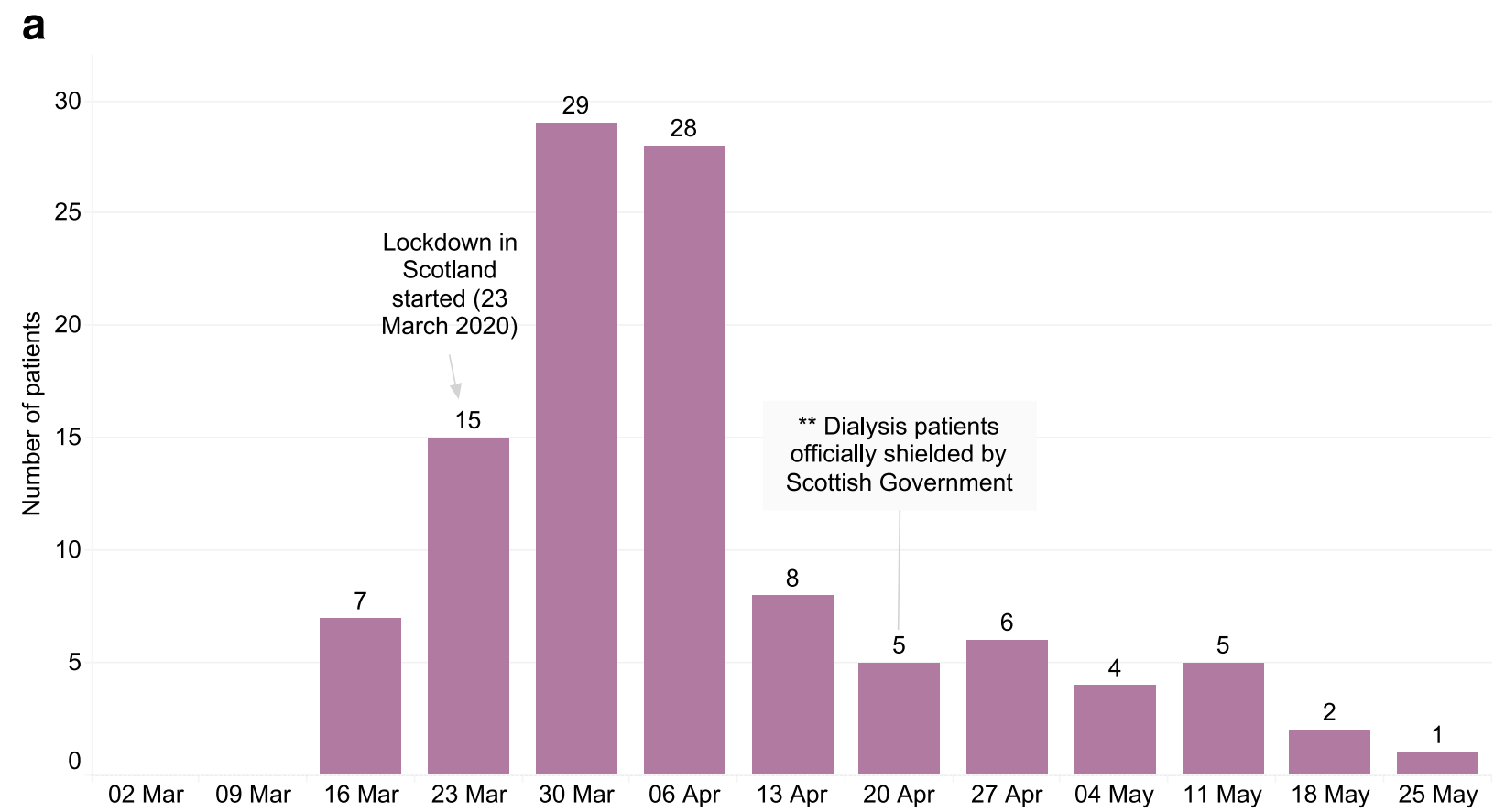

*If a patient has been tested multiple times, the date of the first positive or first negative test was used. If a patient has tested positive, then subsequently tested negative they would only appear in the positive cohort.

${ }^{* *} \mathrm{~A}$ large proportion of patients had been added to the shielding list prior to this point by their care team.

SARS-CoV-2 Positive

b

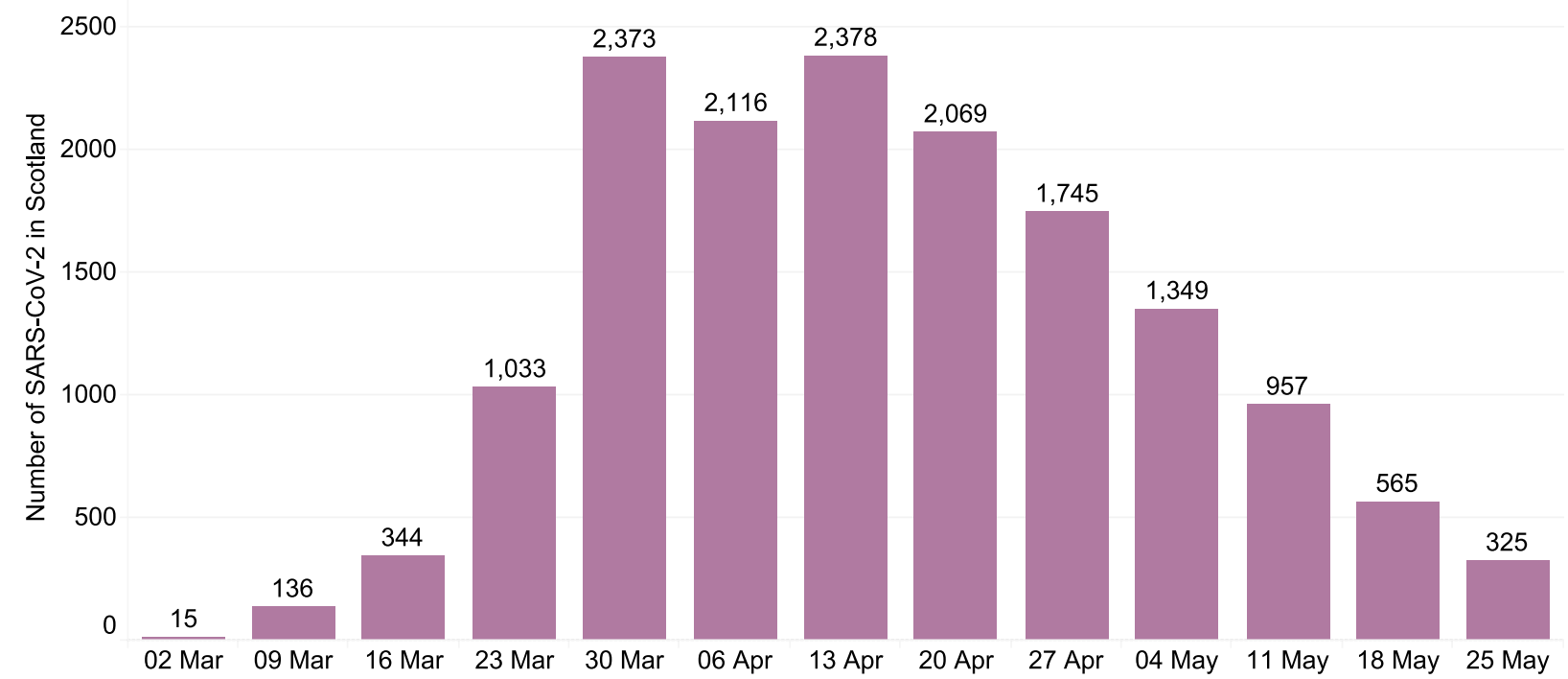

Fig. 3 a: Number of patients* on KRT who tested positive for SARS-CoV-2. *If a patient has been tested multiple times, the date of the first positive or first negative test was used. If a patient has tested positive, then subsequently tested negative they would only appear in the positive cohort. b: Number of patients in Scotland who tested positive for SARS-CoV-2

infection. Mortality was associated with increasing age in this cohort [23]. A study from a single urban centre in the United Kingdom showed much higher rates of transmission with over $20 \%$ of their haemodialysis patients affected within 6 weeks of detection of their first case [24]. Similar mortality rates was demonstrated in this series with $20.3 \%$ patients dying during follow up, again with increasing age being associated with higher 

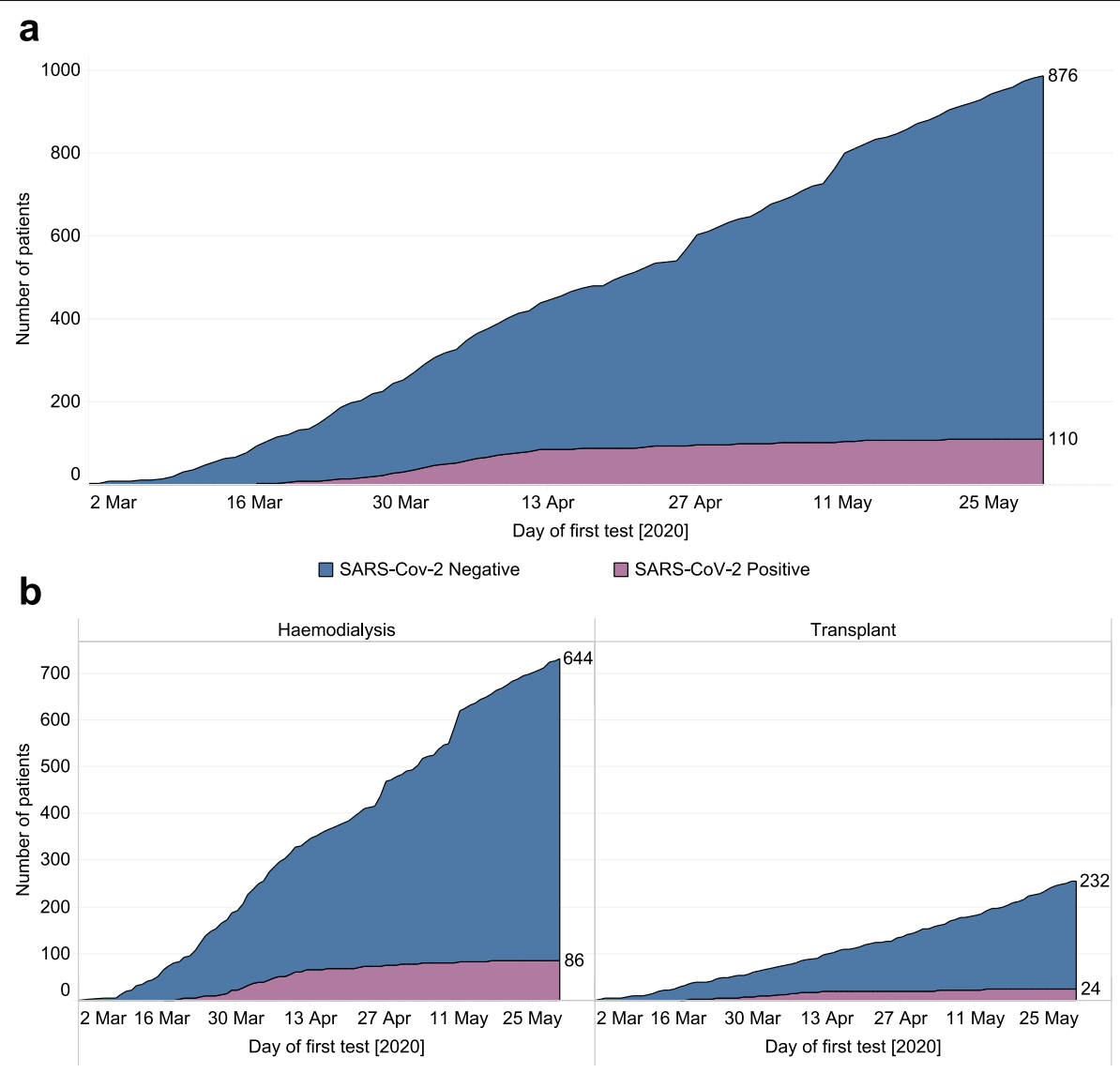

*If a patient has been tested multiple times, the date of the first positive or first negative test was used. If a patient has tested positive, then subsequently tested negative they would only appear in the positive cohort.

\section{$\square$ SARS-Cov-2 Negative $\quad \square$ SARS-CoV-2 Positive}

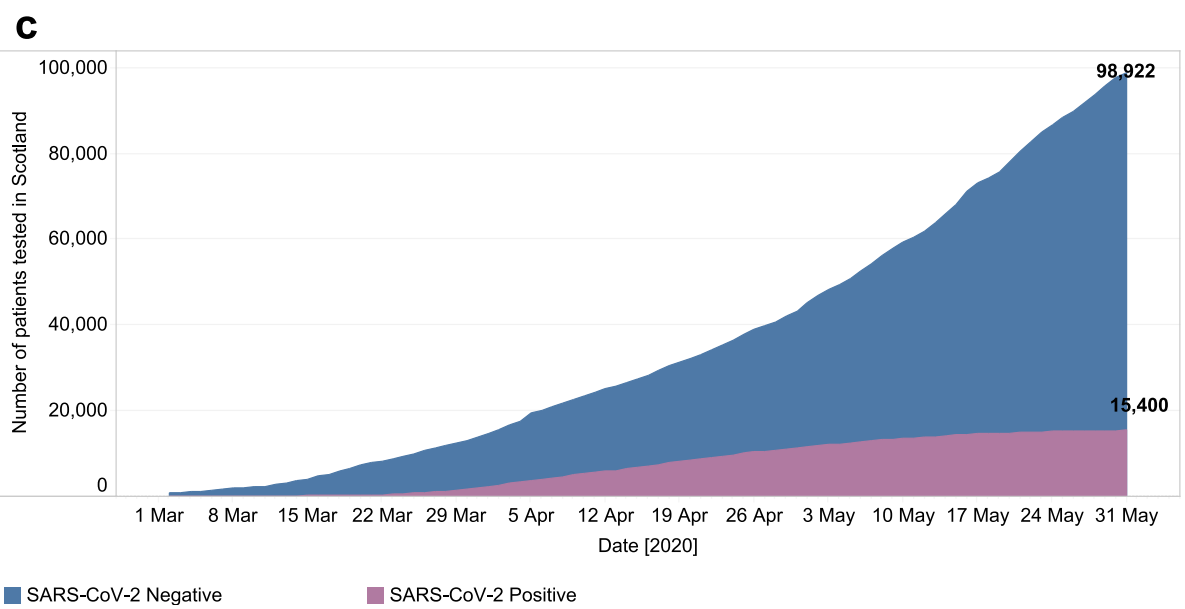

Fig. 4 a: Cumulative number of SRR patients* on KRT with a SARS-CoV-2 test. $\mathbf{b}$ : Cumulative number of SRR patients* on KRT with a SARS-CoV-2 test split by modality. c: Cumulative number of patients in Scotland with a SARS-CoV-2 test (Source: http://www.gov.scot/publications/coronaviruscovid-19-trends-in-daily-data/)

likelihood of mortality. The outcomes we report in kidney transplant recipients with $29.2 \%$ of those with COVID-19 dying is similar to that reported in New York [25]. The number of patients in our cohort who required intensive care is perhaps lower than might be expected. However, within renal units, detection of SARS-COV2 triggered treatment escalation planning. This included discussion with patients about appropriate and realistic ceilings of treatment. As a result the number of people referred to ICU will have been reduced. 
Table 1 Descriptive characteristics of all prevalent patients undergoing KRT in Scotland on 31st May 2020

\begin{tabular}{|c|c|c|c|c|c|}
\hline & & Dialysis & & Transplant & \\
\hline & & $\begin{array}{l}\text { COVID-19+ } \\
n=86\end{array}$ & $\begin{array}{l}\text { Total on HD \& PD } \\
n=2184\end{array}$ & $\begin{array}{l}\text { COVID-19+ } \\
n=24\end{array}$ & $\begin{array}{l}\text { Total Transplant } \\
n=3277\end{array}$ \\
\hline & & n (\%) & n (\%) & n (\%) & n (\%) \\
\hline Sex & Female & $39(45)$ & $879(40)$ & $11(46)$ & $1318(40)$ \\
\hline & Male & $47(55)$ & $1305(60)$ & $13(54)$ & $1959(60)$ \\
\hline Age Group & $20-44$ & $10(12)$ & $244(11)$ & $4(17)$ & $774(24)$ \\
\hline & $45-64$ & $25(29)$ & $829(38)$ & $12(50)$ & $1745(53)$ \\
\hline & $65-74$ & $24(28)$ & $599(27)$ & $5(21)$ & $612(19)$ \\
\hline & $75+$ & $27(31)$ & $512(23)$ & $3(13)$ & $146(4)$ \\
\hline Primary Renal Diagnosis & Glomerulonephritis & $14(16)$ & $374(17)$ & $3(13)$ & $851(26)$ \\
\hline & Interstitial & $20(23)$ & $464(21)$ & $12(50)$ & $1275(39)$ \\
\hline & Multisystem & $15(17)$ & $489(22)$ & $3(13)$ & $386(12)$ \\
\hline & Diabetes & $29(34)$ & $569(26)$ & $3(13)$ & $386(12)$ \\
\hline & Unknown & $8(9)$ & $288(13)$ & $3(13)$ & $379(12)$ \\
\hline${ }^{a}$ Dialysis Vintage & $<1$ year & $20(23)$ & $444(20)$ & $0(0)$ & $310(9)$ \\
\hline & $1-2$ years & $14(16)$ & $349(16)$ & $2(8)$ & $314(10)$ \\
\hline & $2-3$ years & $8(9)$ & $293(13)$ & $1(4)$ & $339(10)$ \\
\hline & $<=5$ years & $22(26)$ & $468(21)$ & $3(13)$ & $698(21)$ \\
\hline & $<=10$ years & $11(13)$ & $243(11)$ & $4(17)$ & $701(21)$ \\
\hline & $>10$ years & $11(13)$ & $301(14)$ & $14(58)$ & $88(27)$ \\
\hline SIMD & $1=$ most deprived & $32(37)$ & $634(29)$ & $7(29)$ & $715(22)$ \\
\hline & 2 & $24(28)$ & $503(23)$ & $5(21)$ & $698(21)$ \\
\hline & 3 & $10(12)$ & 417 (19) & $4(17)$ & $631(19)$ \\
\hline & 4 & $13(15)$ & $356(16)$ & $5(21)$ & $646(20)$ \\
\hline & $5=$ least deprived & $7(8)$ & $274(13)$ & $3(13)$ & $587(18)$ \\
\hline
\end{tabular}

Patients with missing data $(n=14)$ not included in total for analysis purposes - none of which were COVID-19+

${ }^{a}$ Dialysis vintage for those on transplant is taken from the date of the transplant, those on dialysis is their time on KRT

In the general population, a number of comorbid conditions such as diabetes, hypertension and obesity have consistently been linked to increased risk of death with COVID-19 [12]. In patients requiring KRT, it is clear that the risk attributable to requirement for KRT is such a strong risk factor for death that it is challenging to identify subgroups of dialysis or kidney transplant recipients at highest risk.
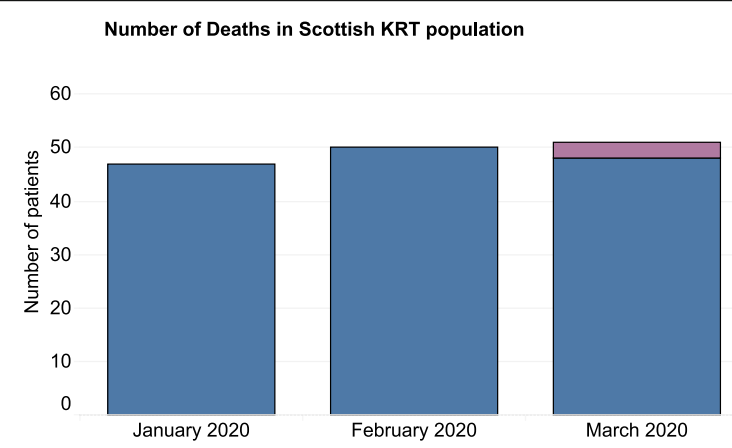

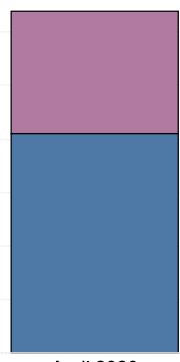

April 2020

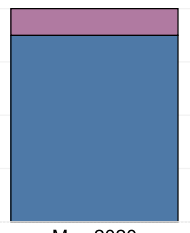

May 2020

*For all those patients who tested positive for SARS-CoV-2 - all had SARS-CoV-2 as Cause of Death on death certificate. $\square$ SARS-CoV-2 Positive $\square$ SRR Population

Fig. 5 Number of Deaths in KRT population per calendar month. *For all those patients who tested positive for SARS-CoV-2 - all had SARS-CoV-2 as Cause of Death on death certificate 


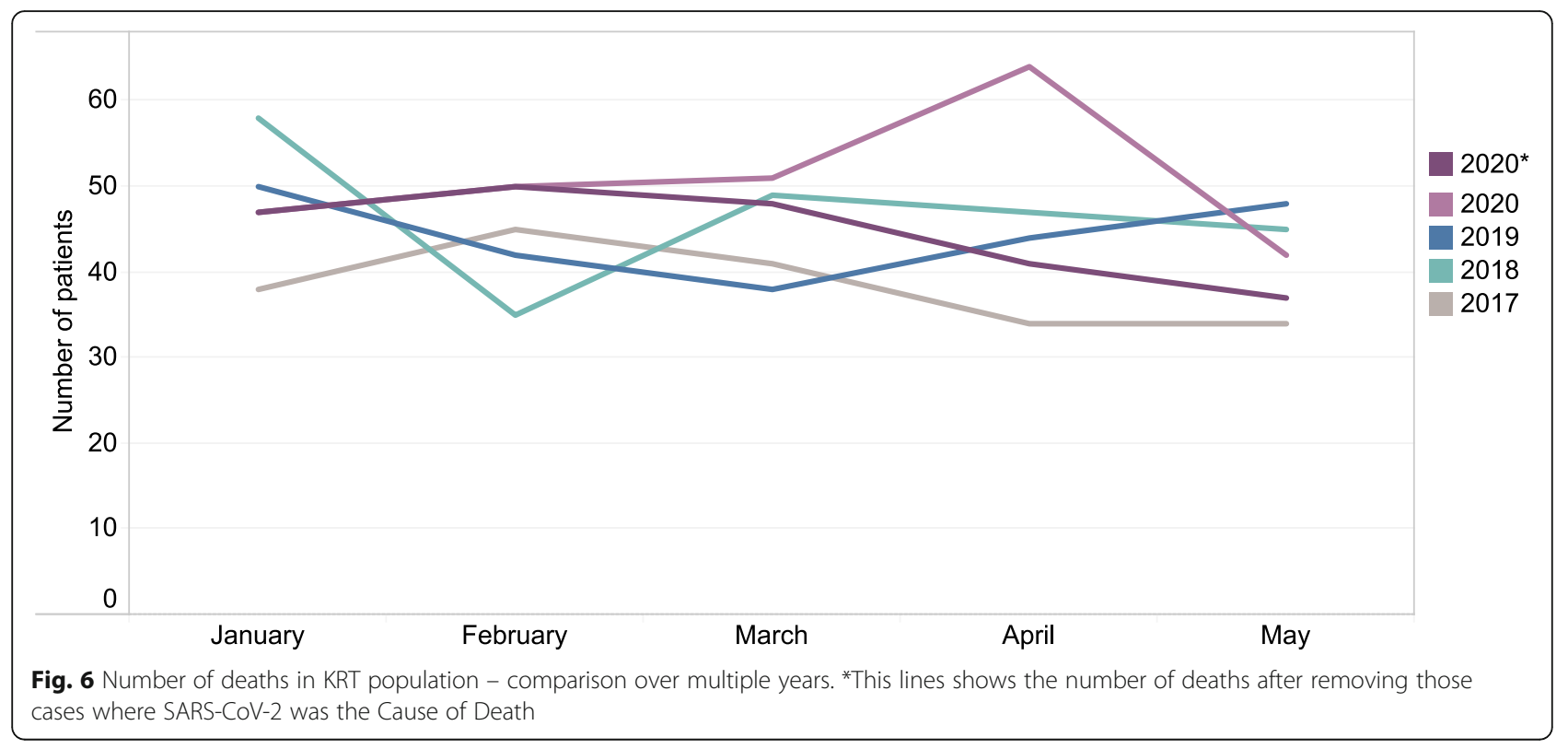

SARS-CoV-2 has affected a relatively small proportion of the KRT patients in Scotland. However, mortality was high in those affected and COVID-19 has been associated with excess mortality in the KRT population of Scotland during this period compared to equivalent periods during previous years. Renal unit staff and transport services have worked hard to mitigate and contain transmission within the individual dialysis and satellite units. The subsequent drop in new cases by the third week of April suggests that these measures coupled with shielding were effective. It is not possible to say with absolute certainty but the experience in a large dialysis unit in London has led to similar conclusions [24]. Infection control measures are likely to have been beneficial in reducing transmission associated with dialysis treatment, either related to transport with other patients, or during treatment itself. However, it is not possible to dissociate the reduction in number of cases of SARS-CoV-2 in the KRT population from the fall observed in the general population in response to measures encompassed by 'lockdown' including closure of schools and nonessential shops, increased hand hygiene and social distancing. However, as the incidence of new SARS-CoV-2 infections specifically in patients on KRT fell prior to any fall in the incidence of SARS-CoV-2 in Scotland (Fig. 3), it is plausible that the combination of measures specific to this high risk group had efficacy in driving down new infections, rather than to simply assume that falling infection in KRT patients reflected reducing community transmission of SARS-CoV-2. It is worth noting that highest numbers of SARS-CoV-2 infection was observed in patients residing in areas of highest socioeconomic deprivation. Although the numbers of infections do not necessarily track to outcomes, most
UK and Scotland data demonstrate that infection rates and deaths with SARS-CoV-2 are higher in areas of socioeconomic deprivation [26].

A strength of our study is that we have comprehensive coverage of all patients receiving KRT in Scotland with linkage to Health Protection Scotland which holds data on all testing for SARS-CoV-2 performed in Scotland. However, limitations of our study are that we did not have data on hospitalisations, co-morbidities, immunosuppression regimes, acute kidney injury or smoking status. Fortunately, our numbers were small. However, this limits our ability to draw conclusions from our analyses. Only symptomatic patients were tested, so we cannot comment on the asymptomatic rate of SARS-CoV-2 infection, this may have led to lower case ascertainment in the population of patients with kidney transplants. However, unlike in the wider UK population, testing was performed liberally, and to the best of our knowledge in all symptomatic patients requiring dialysis and in those without symptoms but who presented with fever.

\section{Conclusion}

In summary, we demonstrate that $2 \%$ of the KRT population had a positive test for SARS-CoV-2 during the period of study. The incidence of positive tests fell following instigation of infection control measures. Mortality is high and it is unclear if any of the therapies demonstrated to improve outcomes in patients with COVID-19 such as dexamethasone or remdesivir will have efficacy in patients requiring KRT. Patients requiring dialysis were excluded from recent remdesivir trials [27]. It is likely that in the absence of specific therapy, and high mortality, heightened vigilance of the risk of SARS-CoV-2 infection, infection control measures including use of PPE for both staff and 


\section{a}

\section{Survival at 30 days from first SARS-CoV-2 test}

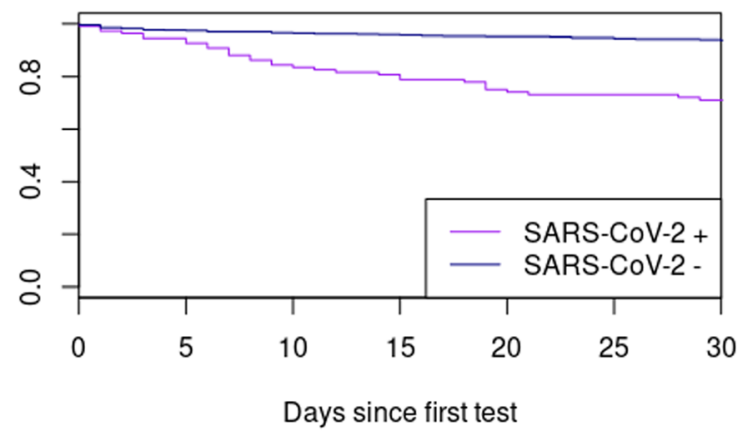

\section{b Survival at 30 days from first SARS-CoV-2 test (Haemodialysis)}

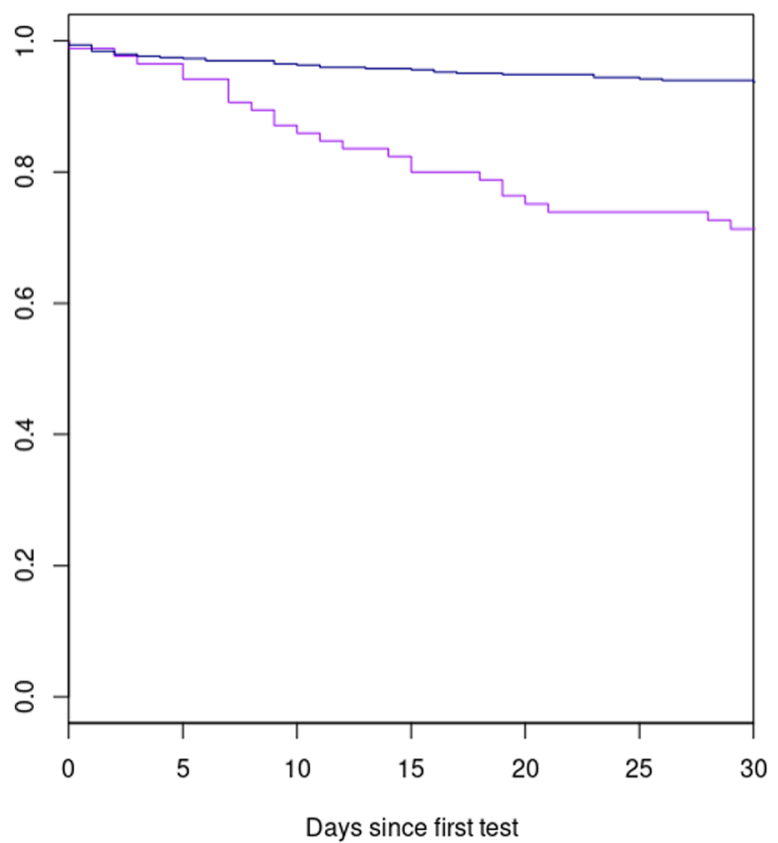

Survival at 30 days from first SARS-CoV-2 test (Transplant)

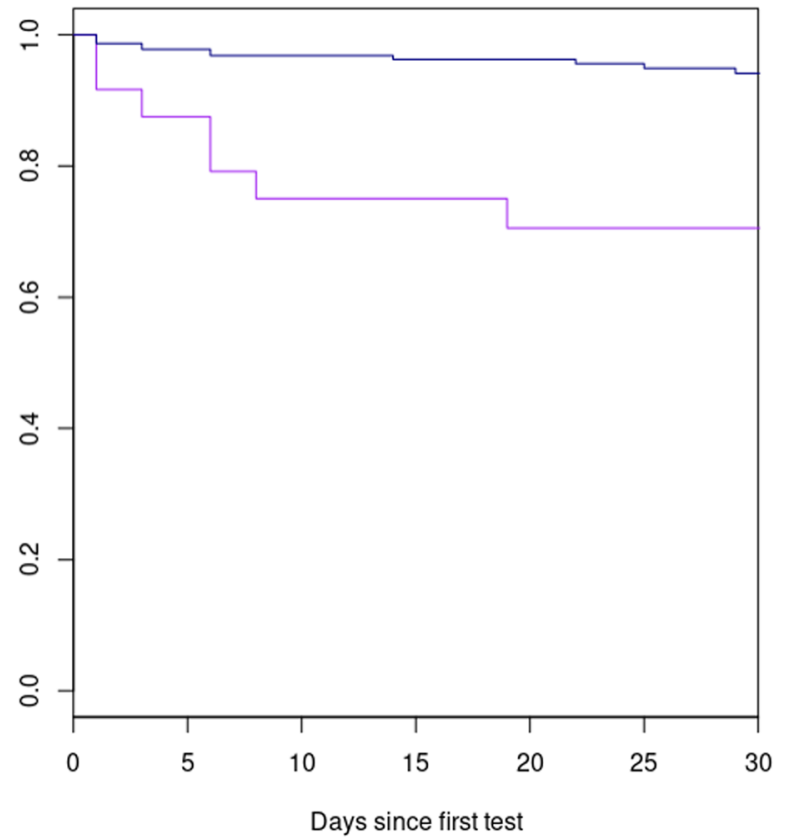

Fig. 7 a: Survival at 30 days from first SARS-CoV-2 test. * Log Rank Test for KM is $p<0.001$. Survival at 30 days: SARS-CoV-2 Neg: 0.91 (95\% Cl 0.894,0.94); SARS-CoV-2 Positive: 0.71 (95\% Cl 0.630, 0.802). b: Survival at 30 days from first SARS-CoV-2 Test split by modality at time of test result. Survival at 30 days Haemodialysis: SARS-CoV-2 Neg: 0.92 (95\% Cl 0.885, 0.94); SARS-CoV-2 Positive: 0.71 (95\% Cl 0.623, 0.817 ). Survival at 30 days Kidney Transplant: SARS-CoV-2 Neg: 0.94 (95\% CI 0.91, 0.98); SARS-CoV-2 Positive: 0.71 (95\% Cl 0.544, 0.915 )

Table 2 Details of ICU/ HDU admission

\begin{tabular}{|c|c|c|c|c|}
\hline & COVID-19 Positive & COVID-19 Negative & Not Tested & Total of SRR Patients \\
\hline Number of patients with ICU/HDU stay & 13 & 77 & 28 & 118 \\
\hline Median Length of Stay & 6.0 & 4.0 & 3.0 & 4.0 \\
\hline Average Advanced Ventilation Days & 2.6 & 0.7 & 0.1 & 0.8 \\
\hline Average Non Invasive Ventilation Days & 0.5 & 0.2 & 0.0 & 0.2 \\
\hline Average Cardio Support Days & 1.4 & 0.7 & 0.2 & 0.7 \\
\hline Average Renal Support Days & 2.8 & 2.1 & 0.5 & 1.8 \\
\hline
\end{tabular}


patients, will be required for the long term to mitigate the risk of SARS-CoV-2 in this vulnerable patient group. This may come at a cost of increasing social isolation and more limited social interaction between other patients, health care workers and wider society. Moreover, the economic cost of providing additional transport for individual patients travelling to dialysis, more dedicated nursing staff and segregated dialysis bays, as well as for additional PPE will add substantially to the existing financial burden of KRT to healthcare systems.

\section{Abbreviations}

SARS-CoV-2: Severe acute respiratory coronavirus 2; KRT: Kidney replacement therapy; CHI: Community health index; ESRD: End stage renal disease; ERAEDTA: European Renal Association-European Dialysis and Transplant Association; NHS BT: National Health Service Blood and Transplant; SIMD: Scottish Index of Multiple Deprivation; SICSAG: Scottish Intensive Care Society Audit Group; RT-PCR: Real-time polymerase chain reaction; SD: Standard deviation; PPE: Personal protective equipment

\section{Acknowledgements}

We thank Kirsty Mangin at Health Protection Scotland and Lorraine Donaldson from SICSAG for their assistance with linkage of the datasets. We thank all staff in critical care units who submitted data to the SICSAG database.

\section{Authors' contributions}

$J C, M O, C W$, JM contributed to the acquisition and analysis of the data. SB and JT were responsible for the conception and design of the work. SB, KB, ZC, MF, NL, SM, RP, ES, WM, PT, AA, VS, PM and JT interpreted the data. All authors drafted the manuscript and approved the final version of the manuscript.

\section{Funding}

No funding was obtained for this work.

\section{Availability of data and materials}

The data underlying this article cannot be shared publicly as they are held by Public Health Intelligence, Scotland. Data can be requested from the electronic Data Research and Innovation Service (eDRIS) team which are part of the Public Health Scotland Public Health Intelligence, Scotland through phs.edris@nhs.net.

\section{Ethics approval and consent to participate}

Ethical approval was deemed unnecessary according to Public Health Scotland Information Governance as this was analysis of routinely collected data. As the analysis used routinely collected and anonymized data, individual patient consent was not sought. Access and use of the data for the purpose of this work were approved following a Public Health Scotland information governance review of linking internal datasets. Only the Public Health Scotland analyst had access to the linked patient data which could only be accessed via an NHS secure network

\section{Consent for publication}

Not applicable.

\section{Competing interests}

PBM is a member of BMC Nephrology Editorial Board.

\section{Author details}

'Division of Population Health and Genomics, School of Medicine, University of Dundee, Dundee DD1 9SY, UK. ${ }^{2}$ The Scottish Renal Registry, Scottish Health Audits, Public Health \& Intelligence, Information Services, Meridian Court, 5 Cadogan Street, Glasgow G2 6QE, UK. ${ }^{3}$ Renal Unit, Victoria Hospital, Kirkcaldy KY2 5AH, UK. ${ }^{4}$ Renal Unit, Monklands Hospital, Monkscourt Avenue, Airdrie ML6 0JS, UK. ${ }^{5}$ Glasgow Renal \& Transplant Unit, Queen Elizabeth University Hospital, Glasgow G51 4TF, UK. ${ }^{6}$ Usher Institute, University of Edinburgh, Teviot Place, Edinburgh EH8 9AG, UK. 'Department of Renal
Medicine, Royal Infirmary of Edinburgh, Edinburgh Bioquarter, Edinburgh EH16 4SA, UK. ${ }^{8}$ Department of Renal Medicine, Aberdeen Royal Infirmary, Foresterhill Health Campus, Foresterhill Rd, Aberdeen AB25 2ZN, UK. ${ }^{9}$ Renal Unit, Raigmore Hospital, Old Perth Road, Inverness IV2 3UJ, UK. ${ }^{10}$ Renal Unit, Mountainhall Treatment Centre, Dumfries DG1 4AP, UK. " Renal Unit, Ninewells Hospital, Dundee DD1 9SY, UK. ${ }^{12}$ Renal Unit, University Hospital Crosshouse, Crosshouse KA2 OBE, UK.

Received: 13 July 2020 Accepted: 9 September 2020

Published online: 01 October 2020

\section{References}

1. Zhu N, Zhang D, Wang W, Li X, Yang B, Song J, et al. A novel coronavirus from patients with pneumonia in China, 2019. N Engl J Med. 2020;382(8): 727-33.

2. Wu Z, McGoogan JM. Characteristics of and important lessons from the coronavirus disease 2019 (COVID-19) outbreak in China: summary of a report of 72314 cases from the Chinese Center for Disease Control and Prevention. JAMA. 2020;323(13):1239-42.

3. Kato S, Chmielewski M, Honda H, Pecoits-Filho R, Matsuo S, Yuzawa Y, et al. Aspects of immune dysfunction in end-stage renal disease. Clin J Am Soc Nephrol. 2008:3(5):1526-33.

4. Basile C, Combe C, Pizzarelli F, Covic A, Davenport A, Kanbay M, et al. Recommendations for the prevention, mitigation and containment of the emerging SARS-CoV-2 (COVID-19) pandemic in haemodialysis centres. Nephrol Dialysis Transplant. 2020;35(5):737-41.

5. Ikizler TA, Kliger AS. Minimizing the risk of COVID-19 among patients on dialysis. Nat Rev Nephrol. 2020;16(6):311-3.

6. Meijers B, Messa P, Ronco C. Safeguarding the maintenance hemodialysis patient population during the coronavirus disease 19 pandemic. Blood Purif. 2020:49(3):259-64

7. Kliger AS, Cozzolino M, Jha V, Harbert G, Ikizler TA. Managing the COVID-19 pandemic: international comparisons in dialysis patients. Kidney Int. 2020; 98(1):12-16

8. UK Renal Association. PPE and use of masks by dialysis patients 2020 [Available from: https://renal.org/renal-association-statement-ppe-use-masksdialysis-patients/\#layout-secondary-nav.

9. Wu J, Li J, Zhu G, Zhang Y, Bi Z, Yu Y, et al. Clinical features of maintenance hemodialysis patients with 2019 novel coronavirus-infected pneumonia in Wuhan. China Clin J Am Soc Nephrol. 2020;15(8):1139-45.

10. Cho JH, Kang SH, Park HC, Kim DK, Lee SH, Do JY, et al. Hemodialysis with cohort isolation to prevent secondary transmission during a COVID-19 outbreak in Korea. J Am Soc Nephrol. 2020;31(7):1398-1408.

11. Valeri AM, Robbins-Juarez SY, Stevens JS, Ahn W, Rao MK, Radhakrishnan J, et al. Presentation and outcomes of patients with ESKD and COVID-19. J Am Soc Nephrol. 2020;31(7):1409-15.

12. Docherty AB, Harrison EM, Green CA, Hardwick HE, Pius R, Norman $L$, et al. Features of 20133 UK patients in hospital with covid-19 using the ISARIC WHO clinical characterisation protocol: prospective observational cohort study. BMJ. 2020;369:m1985. https://doi.org/10.1136/bmj.m1985.

13. Wang D, Hu B, Hu C, Zhu F, Liu X, Zhang J, et al. Clinical Characteristics of 138 Hospitalized Patients With 2019 Novel coronavirus-infected pneumonia in Wuhan, China. JAMA. 2020;323(11):1061-69.

14. Filipe ADS, Shepherd J, Williams T, Hughes J, Aranday-Cortes E, Asamaphan $\mathrm{P}$, et al. Genomic epidemiology of SARS-CoV-2 spread in Scotland highlights the role of European travel in COVID-19 emergence. medRxiv. 2020;2020:06. 08.20124834.

15. Scottish Renal Registry. ERA-EDTA Primary Renal Diagnosis Codes, and Groupings used in SRR analyses [Available from: https://www.srr.scot.nhs.uk/ Projects/Methods.html\#int].

16. Public Health Scotland. The Scottish Index of Multiple Deprivation (SIMD) [Available from: https://www.isdscotland.org/Products-and-Services/GPDSupport/Deprivation/SIMD/].

17. Public Health Scotland. The Scottish Intensive Care Audit Group [Available from: https://www.sicsag.scot.nhs.uk/index.html].

18. R Core Team. R: A Language and Environment for Statistical Computing [Internet] Vienna, Austria 2008 [Available from: http://www.r-project.org].

19. Scottish Government. Coronavirus (COVID-19): clinical guidance for managing patients; 2020. [Available from: https:/www.gov.scot/ publications/coronavirus-covid-19-clinical-advice/]. 
20. Wang R, Liao C, He H, Hu C, Wei Z, Hong Z, et al. COVID-19 in hemodialysis patients: a report of 5 cases. Am J Kidney Dis. 2020;76(1):141-3.

21. Alberici F, Delbarba E, Manenti C, Econimo L, Valerio F, Pola A, et al. A report from the Brescia renal COVID task force on the clinical characteristics and short-term outcome of hemodialysis patients with SARS-CoV-2 infection. Kidney Int. 2020;98(1):20-26.

22. Goicoechea M, Sánchez Cámara LA, Macías N, Muñoz de Morales A, Rojas ÁG Bascuñana A, et al. COVID-19: clinical course and outcomes of 36 hemodialysis patients in Spain. Kidney International. 2020;98(1):27-34.

23. Sánchez-Álvarez JE, Pérez Fontán M, Jiménez Martín C, Blasco Pelícano M, Cabezas Reina CJ, Sevillano Prieto ÁM, et al. Situación de la infección por SARS-CoV-2 en pacientes en tratamiento renal sustitutivo. Informe del Registro COVID-19 de la Sociedad Española de Nefrología (SEN). Nefrología. 2020;40(3):272-78.

24. Corbett RW, Blakey S, Nitsch D, Loucaidou M, McLean A, Duncan N, et al. Epidemiology of COVID-19 in an Urban Dialysis Center. J Am Soc Nephrol. 2020;31(8):1815-23.

25. Akalin E, Azzi Y, Bartash R, Seethamraju H, Parides M, Hemmige V, et al. Covid-19 and kidney transplantation. N Engl J Med. 2020;382(25):2475-7.

26. Williamson E, Walker AJ, Bhaskaran KJ, Bacon S, Bates C, Morton CE, et al. Factors associated with COVID-19-related death using OpenSAFELY. Nature. 2020:584(7821):430-36.

27. Beigel JH, Tomashek KM, Dodd LE, Mehta AK, Zingman BS, Kalil AC, et al. Remdesivir for the treatment of Covid-19 - preliminary report. N Engl J Med. 2020:NEJMoa2007764. https://doi.org/10.1056/NEJMoa2007764.

\section{Publisher's Note}

Springer Nature remains neutral with regard to jurisdictional claims in published maps and institutional affiliations.

Ready to submit your research? Choose BMC and benefit from:

- fast, convenient online submission

- thorough peer review by experienced researchers in your field

- rapid publication on acceptance

- support for research data, including large and complex data types

- gold Open Access which fosters wider collaboration and increased citations

- maximum visibility for your research: over $100 \mathrm{M}$ website views per year

At $\mathrm{BMC}$, research is always in progress.

Learn more biomedcentral.com/submissions 